\title{
The Influence of Leadership style on teachers' job satisfaction in Public Universities in Kazakhstan
}

Gulnar Orynbek ${ }^{1 *}$, Xu Hui ${ }^{2}$, Kalibekuly Tolkyn ${ }^{3}$, Endale Fantahun Tadesee ${ }^{4}$, Sabika Khalid $^{5}$

${ }^{1} \mathrm{PhD}$ candidate for Educational Leadership and Management, Collage of Education, Southwest University China, CHINA

${ }^{2}$ Professor. Dr., Collage of Education, Southwest University China, CHINA

${ }^{3}$ Candidate's PhD, Associate professor of Linguistics, Kazakh Anlai Khan University of International Relations and World Languages

${ }^{4} \mathrm{PhD}$ candidate for Educational Leadership and Management, Collage of Education, Southwest University China, CHINA

${ }^{5} \mathrm{PhD}$ candidate for Educational Leadership and Management, Collage of Education, Southwest University China, CHINA

*Corresponding author

Email: nara.kz@,bk.ru (Gulnar Orynbek), skcxh@,swu.edu.cn (Xu Hui)

\section{Abstract}

This study was examined the relationship between faculty leaders' leadership style and faculty teachers' job satisfaction at two public universities in Kazakhstan (L.N.Gomylov Eurasian National University, Kazakh Women Teacher Training University). One hundred twenty-four academic staff participated the research (117 teachers, 6 faculty leaders), completed the Multifactor leadership Questionnaire (MLQ) and Mohrman-Cook-Mohrman 
Job satisfaction Scale (MCMJSS). The collection of data is supporting the idea of there is a strong correlation between leaders' leadership style and teachers job satisfaction. Transactional leadership style is the most used by faculty leaders (Dean, HOD) in these two universities. Transformational, Transactional leadership style have positive impact on teachers' job satisfaction level, while the Laisses-faire leadership style has negative influence. Another finding is showed that female teachers tend to be more satisfied than male teachers, the teachers with lower qualification are more satisfied than the $\mathrm{PhD}$ holders and professors. same result can be seen in other scholars' findings (Sharma \& Jyoyi, 2009; Wagner \& French, 2010).

Keywords: leadership style, teachers, job satisfaction, transformational, transactional, laisses-faire leadership style.

\section{Background of the study}

Teachers can be considered most important professional group in future education filed, and many teachers not really satisfied about their job according to Ingersoll 2003. One of the most leading factor which affects for teachers job satisfaction is the leadership style of administrators (Ingersoll, 2003; Zahari \& Shurbagi, 2012). Furthermore, earlier retired teachers who are dissatisfied with their job usually (Ingersoll, 2003) and abandon the teaching profession, increasing teacher shortages. Teachers job satisfaction level can develop culture as well as the success of the students' level (Dale, 2012).

Teacher' satisfaction from the job is highly important for the nexus between teachers and students, for satisfied teachers will be more enthusiastic about investing time and energy in teaching their students (Bogler, 2001:679; Sharma and Jyoti, 2009). An appropriate leadership style is more likely to enhance job satisfaction among the teachers/faculty (Fowler, 1991), to potentially increase their performance (Madlock, 2008), and consequently to achieve institutional success (Ngui et al., 2006). satisfied workers are more efficient and effective in an organization (Haque \& Aston, 2016; Haque et al., 2015). Subordinates will be more satisfied if they are treated well (Aziri, 2011; Haque, Faizan \& Cockrill, 2017). Individuals' job satisfaction level is visible from their attitude towards their work. Highly 
satisfied employees have a positive and favorable attitude towards their work while unsatisfied workers have a negative attitude towards their job (Armstrong, 2006).

\section{Statement of the Problem}

Kazakhstan is located in central Asia with the population of 18 million (2018). There are 177 universities, 68 public and 109 privates. Universities have different outcomes within the same city even in same departments. Teachers' job satisfaction is the one very important key factor influencing faculty achievement. There are number of factors influencing teachers job satisfaction level such as school environment, salary, benefits, teacher placement and leadership style. Existing studies show that leaders' leadership style is the main motivation for the teachers (Barling et al, 2002; Vivian Robinson, 2007). However, in Higher education context there are very less studies have worked on this topic in Kazakhstan.

In University level academic dean has key roles within the institutions, (McGregor, 2005) mentioned that there is little or no formal leadership training for the academic leaders in University because many deans rise from the ranks of faculty to the deanship position. Academic dean play the role as collage leader, university representative, consensus builder, mediator, and facilitator (Rosser, Johnsrud, \& Heck, 2003). In University level academic dean has direct impact for the teachers job satisfaction and organizational success. Leary et al. (1999) investigated the relationship between leadership styles and faculty job satisfaction in the higher education context of the United States. The study focuses on collage level, and faculty members rated the leadership styles of their leaders and their job satisfaction. The findings demonstrated that generally there was a statistically significant relationship between leadership styles and the faculty's overall job satisfaction.

\section{○ Transformational leadership}

Transformational leaders motivate subordinates in inspirational and fetch changes in an organization (Burns, 1978; Webeer, 2009). Last research states this leadership style improve the performance of organization (Weber, 2009). According to Riggio (2006) transformational leadership style follows: 1 . The inspirational motivation dimensions. 2 . The idealized influence dimension. 3. The intellectual stimulation dimension. 4. The individual consideration dimension (Bass \& Riggio, 2006). According to Weistein (2004), transformational leaders are concerned about the well-being employees. This leadership style encourages their subordinates and work with each individuals' need to be success. Avolio 
and Bass (1995) states that transformational leaders let their employees to follow by setting positive examples as well as set high expectations, and let them to think outside of the box.

\section{$\Rightarrow \quad$ Transactional leadership}

Transactional leaders motivate their subordinates by giving reward and punishment. Transformational leadership has three different style: Contingent reword, Management-byException (active and passive ) (Bass, 1998; Gill, 2006).

\section{$\Rightarrow \quad$ Laisses-Faire leadership}

Laisses-faire leadership is characterized as non-leadership or the absence of leadership. (Avolio et al., 1999; Northouse, 2010).

\section{Factors affecting Teachers' Job Satisfaction}

Scholars have found out that there are different factors influence teachers job satisfaction level (Collie et al., 2012; Klassen \& Anderson, 2009; Klassen \& Chiu, 2010; Moe et al., 2010). We can divide these factors in to three categories: the factor which is student-related, the factor which is related instructor, and the factor which is related by institution.

Student-related factors- studies have found out that there is a positive relation between students performance and teachers job satisfaction (Sloan Consortium, 2006). In the school context if the students make improvement in their study or if they perform good, the teachers job satisfaction level also getting high (Fredericksen, Pickett, Shea, Pelz, \& Swan, 2000).

\section{Factors affecting Teachers' Job Satisfaction}

Researchers have found out that there are some factors which have influence for teachers job satisfaction level

(Collie et al., 2012; Klassen \& Anderson, 2009; Klassen \& Chiu, 2010; Moe et al., 2010). We can divide these factors in to three categories: the factor which is student-related, the factor which is related instructor, and the factor which is related by institution.

Student-related factors- according to studies there is a connection between students performance and teachers job satisfaction (Sloan Consortium, 2006). Teachers would be more satisfied if their students perform well (Fredericksen, Pickett, Shea, Pelz, \& Swan, 2000).

Instructor-related factors- teachers' job satisfaction can be very different from each other. Panda \& Mishra (2007) states that teachers academic experience is an important factor while 
other researchers found that self-gratification (Rockwell, Schauer, Fritz, \& Marx, 1999). Academic performance of teachers has direct impact to their overall job satisfaction (Cockburn, 2000), teachers' preparation for their job is widely different, some of them have in-service training with good mentor that give them careful guiding, when others enter the organization without any experiences of training. Teachers with high level of academic preparation, be able to deliver knowledge more effectively in the class are tend to be more satisfied (higher level of satisfaction on their job) than others. There are two different contradiction idea about the academic performance of teachers on their satisfaction level of job, some scholars believes that better-qualified teachers are dissatisfied and likely to leave from organization than the less qualified teachers (Klassen \& Anderson, 2009). This maybe the result of better qualified teachers easy to find out better job. On the other hand the teacher shortage cause the employment of uncertified teachers and these teachers monthly income is lower than qualified teachers (Klassen \& Chiu, 2010), which also affect their job satisfaction level.

Demographic characteristics - According to the study some demographic characteristics of teachers also have influence for teacher job satisfaction. Most of the present studies showed that there is a correlation between gender and teachers job satisfaction level (August \& Waltman, 2004; Bilimoria et al., 2006; Callister, 2006; Hagedorn, 2000; Hult, Callister, \& Sullivan, 2005; Okpara, Squillance, \& Erondu, 2005; WaRD \& Slooane, 2000), scholars findings showed that male teachers tend to be more satisfied than female teachers (Bilimoria et al., 2006; Callister, 2006; Hult et al., 2005; Olsen et al., 1995; Settles et al., 2006; Tack \& Patitu, 1992), others have found opposite (Sharma \& Jyoti, 2009; Wagner \& French, 2010). Except gender differences scholars also identified that the teacher with young age are less satisfied and more likely to leave from their working places than alders (Viel-Ruma et al., 2010). Marital status is can be also consideration for the correlation between teacher job satisfaction level. According to studies marriage has positive relation with teacher job satisfaction (Sabharwal \& Corley, 2009; Sharma \& Jyoti, 2009). A study about teacher organizational commitment at secondary School in Addis Ababa showed that there is a correlation between teachers' marital stage and their commitment level and conclude that marriage teachers are more committed than single teachers (Endale, 2019). Noordin \& Jusoff (2009) said that married women over 40 years of age were the most satisfied teachers. 


\section{Methodology}

\section{Research Questions}

1. What kinds of leadership style the Faculty leaders (Dean) are using?

2. What is the teachers' perception of job satisfaction in two public universities in Kazakhstan?

3. What is the relationship between faculty leaders' leadership style and teachers job satisfaction?

\section{Sampling}

The study population in this study was 124, in two public universities in Kazakhstan, including 117 teachers and 7 leaders.

Table 1 Ethnographic Description of the Participants

\begin{tabular}{|c|c|}
\hline \multicolumn{2}{|c|}{ University } \\
\hline ENU & $67 \%$ \\
\hline WNU & Gender \\
\hline \multicolumn{2}{|c|}{$18 \%$} \\
\hline Male & $82 \%$ \\
\hline Female & Age \\
\hline \multicolumn{2}{|c|}{} \\
\hline Less than 30 & $24 \%$ \\
\hline $31-40$ & $43 \%$ \\
\hline $41-50$ & $20 \%$ \\
\hline Above 50 & \\
\hline
\end{tabular}

\begin{tabular}{|l|c|}
\hline \multicolumn{2}{|c|}{ Educational level } \\
\hline Master & $68 \%$ \\
\hline PhD & $27 \%$ \\
\hline Professor & $6 \%$ \\
\hline \multicolumn{2}{|c|}{ Marital states } \\
\hline Married & $47 \%$ \\
\hline Unmarried & $53 \%$ \\
\hline \multicolumn{2}{|c|}{ Year of Experience } \\
\hline $1-5$ years & $29 \%$ \\
\hline $5-10$ years & $22 \%$ \\
\hline $10-15$ years & \\
\hline Above 15 & \\
\hline
\end{tabular}

ENU=L.N Gumilyov Eurasian National University, WNU=Kazakh national women's teacher training University

As shown in table 1, the inconsistency in the participants number according to gender, year of experiences are not based on the selectively of the researcher; rather, it is based on the availability of the participants in the faculty of university.

\section{2. research instrument}

In this study, the research administered questionnaires to collected data from 124 participants from two public universities in Kazakhstan. 


\section{Findings and discussions}

The purpose of this study is that to find out the what kinds of leadership style the universities leaders are using, also analysis the relationship between leadership style of university faculty leaders and teachers job satisfaction.

\section{What kinds of leadership style the Faculty leaders (Dean) are using?}

The responses for MLQ (Bass \& Avolio, 2004) were all within the range of 0 to 4, table 2 shows that the faculty leaders from two universities use Transactional leadership styles, Transformational leadership style (Mean=3.3775, $\mathrm{SD}=0.676)$, followed by Transactional leadership style (Mean=2.7, SD=0.62), and Laisses-faire-leadership style (Mean=2.65, $\mathrm{SD}=0.63)$.

Table 2 leaders leadership style

\begin{tabular}{cccccc}
\hline & $\mathrm{N}$ & Minimum & Maximum & Mean & Std. Deviation \\
\hline $\begin{array}{c}\text { Transformational-leadership } \\
\text { style }\end{array}$ & $\mathbf{1 2 3}$ & 1.00 & 5.25 & 2.8085 & 0.63879 \\
& & & & & \\
& & & & & \\
\hline Transactional-leadership style & $\mathbf{1 2 3}$ & 1.83 & 5.00 & 3.3775 & 0.67608 \\
\hline Laisses-faire-leadership style & $\mathbf{1 2 3}$ & 0.00 & 4.00 & 2.6531 & 0.64217 \\
\hline
\end{tabular}

$\mathrm{N}=$ number

Table 3 leaders perspective of their leadership style

\begin{tabular}{cccccc}
\hline & N & Minimum & Maximum & Mean & Std. Deviation \\
\hline Transformational & 7 & 2.67 & 3.50 & 3.0714 & 0.34503 \\
\hline Transactional & 7 & 2.67 & 3.83 & 3.1905 & 0.39002 \\
\hline Laisses-faire & 7 & 2.62 & 3.62 & 3.0204 & 0.33656 \\
\hline
\end{tabular}

$\mathrm{N}=$ Number

The leaders $(\mathrm{N}=7)$ from two universities believed that they are using transactional leadership style with (Mean=3.1905, $\mathrm{SD}=0.39$ ), transformational leadership style (Mean=3.0714, $\mathrm{SD}=0.34503)$, Laissses-faire style (Mean=3.0204, $\mathrm{SD}=0.33656)$.

\section{Is there any correlation between faculty deans' leadership style and teacher job} satisfaction?

The study has showed that there is a significant relationship between teachers' job satisfaction and faculty dean's leadership (Transformational, Transactional, Lassis-fare) style. 
The results suggest that there is a significant positive and negative relationship between teachers' job satisfaction and transactional leadership style in Kazakhstan. The more leader is using transactional leadership style, the high satisfaction he/she would have regarding payment $(\mathrm{r}=0.484$, $\mathrm{Sig}=0.000)$, promotion $(\mathrm{r}=0.601$, $\mathrm{Sig}=0.000)$, if the leader is using the transactional leadership style the teacher job satisfaction for coworker $(\mathrm{r}=-0.67, \mathrm{Sig}=0.000)$, nature of work $(\mathrm{r}=-0.020, \mathrm{Sig}=0.833)$ is low, another words the teachers are not satisfied with the nature of work and coworkers if the leader is using transactional leadership style. There are also positive and negative correlation with transformational leadership style and teachers job satisfaction level, but they are very weak. Such as the employees satisfaction with payment $(r=0.057)$, Promotion $(r=-0.055)$, Supervision $(r=0.130)$, fringe benefits $(r=0.006)$, contingent rewords $(\mathrm{r}=0.083)$, communication $(\mathrm{r}=0.121)$.

Table 4 Correlations

\begin{tabular}{|c|c|c|c|}
\hline & Transformational & Transactional & Laisses-faire \\
\hline \multirow{2}{*}{$\begin{array}{c}\text { Pay } \\
\text { Pearson correlation } \\
\text { Sig }\end{array}$} & 0.057 & 0.484 & 0.107 \\
\hline & 0.546 & 0.000 & 0.251 \\
\hline \multirow{2}{*}{$\begin{array}{c}\text { Promotion Pearson correlation } \\
\text { Sig }\end{array}$} & -0.055 & 0.601 & -0.089 \\
\hline & 0.560 & 0.000 & 0.340 \\
\hline \multirow{2}{*}{$\begin{array}{l}\text { Supervision Pearson correlation } \\
\text { Sig }\end{array}$} & 0.130 & 0.299 & -0.009 \\
\hline & 0.165 & 0.001 & 0.921 \\
\hline \multirow{2}{*}{$\begin{array}{l}\text { fringebenefits }{ }^{\text {Pearson correlation }} \\
\qquad \mathrm{Sig}\end{array}$} & 0.006 & 0.581 & 0.035 \\
\hline & 0.948 & 0.000 & 0.707 \\
\hline \multirow{2}{*}{$\begin{array}{c}\text { Contingentrewords }{ }^{\text {Pearson correlation }} \\
\text { Sig }\end{array}$} & 0.083 & 0.494 & 0.104 \\
\hline & 0.376 & 0.000 & 0.267 \\
\hline \multirow{2}{*}{$\begin{array}{l}\text { operatingprocedires }{ }^{\text {Pearson correlation }} \\
\text { Sig }\end{array}$} & -0.25 & 0.609 & 0.022 \\
\hline & 0.791 & 0.000 & 0.816 \\
\hline \multirow{2}{*}{$\begin{array}{l}\text { Coworkers }{ }^{\text {Pearson correlation }} \\
\text { Sig }\end{array}$} & 0.145 & -0.067 & -0.66 \\
\hline & 0.119 & 0.472 & 0.482 \\
\hline \multirow{2}{*}{$\begin{array}{l}\text { Nature of work }{ }^{\text {Pearson correlation }} \\
\text { Sig }\end{array}$} & 0.183 & -0.20 & -0.220 \\
\hline & 0.049 & 0.833 & 0.018 \\
\hline \multirow{2}{*}{$\begin{array}{l}\text { Communication Pearson correlation } \\
\text { Sig }\end{array}$} & 0.121 & 0.256 & 0.066 \\
\hline & 0.197 & 0.005 & 0.479 \\
\hline
\end{tabular}

$(-1<\mathrm{R}<1, \mathrm{P}>0.5)$ 


\section{Research question}

What is the teacher's perception of job satisfaction in two public universities in Kazakhstan?

As it can be seen in table 3 , in 9 areas of teachers' satisfaction are relatively high. At the scale from 0 to 6 , they all over the vale 3 . However, the highest satisfaction is coworkers, the teachers are more satisfied with their colleges. After that, there are nature of work (Mean=3.9, $\mathrm{SD}=0.72$ ), communication (Mean=3.8, $\mathrm{SD}=0.85$ ), supervision (Mean=3.79, $\mathrm{SD}=0.82$ ). as we can see in table 5 the total of teachers' satisfaction with their job is not high (Mean=3.6, $\mathrm{SD}=0.43$ ). it should be noted that there is very small scope of areas with highest and lowest level of satisfaction. As we can see in table 4 the total of teachers' satisfaction is relatively high $(\mathrm{M}=3.67)$

Table 5 the descriptive of the teachers' job satisfaction areas

\begin{tabular}{cccccc}
\hline & $\mathrm{N}$ & Min & Max & M & SD \\
\hline Pay & 117 & 1.00 & 5.25 & 3.1282 & .82041 \\
\hline Promotion & 117 & 2.00 & 5.75 & 3.4915 & .77885 \\
\hline Supervision & 117 & 1.00 & 6.00 & 3.7970 & .81865 \\
\hline Fringe benefit & 117 & 1.50 & 5.00 & 3.4124 & .75238 \\
\hline Contingent rewords & 117 & 1.75 & 5.50 & 3.6735 \\
\hline Operating & 117 & 1.50 & 5.75 & & .16136 \\
procedures & & & & 4.1239 & .72265 \\
\hline Coworkers & 117 & 2.50 & 6.00 & 3.9701 & .73561 \\
\hline Nature of work & 117 & 2.00 & 5.75 & 3.8675 & .85448 \\
\hline communication & 117 & 2.25 & 6.00 & & \\
\hline
\end{tabular}

$\mathrm{N}=$ number of respondents, Min=Minimum, Max=Maximum, $\mathrm{SD}=$ standard deviation

Table 6 Job-satisfaction table

\begin{tabular}{llcccc}
\hline & $\mathrm{N}$ & $\min$ & $\max$ & $\mathrm{M}$ & $\mathrm{SD}$ \\
\hline Job-Satisfaction & 117 & 2.72 & 4.94 & 3.6717 & 0.43777 \\
\hline
\end{tabular}

$\mathrm{N}=$ number of respondents, Min=Minimum, Max=Maximum, $\mathrm{SD}=$ standard deviation 
Table 7 Teachers job satisfaction level in two universities

\begin{tabular}{|c|c|c|c|c|c|}
\hline University & $\mathrm{N}$ & & Mean & Std & Std. Error Mean \\
\hline \multirow[t]{2}{*}{ Pay } & ENU & 51 & 3.1814 & .85744 & .12007 \\
\hline & WNU & 65 & 3.0962 & .79757 & .09893 \\
\hline \multirow[t]{2}{*}{ Promotion } & ENU & 51 & 3.4069 & .80461 & .11267 \\
\hline & WNU & 65 & 3.5423 & .75463 & .09360 \\
\hline \multirow[t]{2}{*}{ supervision } & ENU & 51 & 3.8873 & .99789 & .13973 \\
\hline & WNU & 65 & 3.7308 & .65148 & .08081 \\
\hline \multirow[t]{2}{*}{ Fringe benefits } & ENU & 51 & 3.2843 & .71428 & .10000 \\
\hline & WNU & 65 & 3.5115 & .77740 & .09643 \\
\hline \multirow[t]{2}{*}{ Contingent rewords } & ENU & 51 & 3.8186 & .82927 & .11612 \\
\hline & WNU & 65 & 3.4192 & .76177 & .09449 \\
\hline \multirow[t]{2}{*}{ Operating procedures } & ENU & 51 & 3.7304 & 1.17988 & .16522 \\
\hline & WNU & 65 & 3.6385 & 1.15754 & .14358 \\
\hline \multirow[t]{2}{*}{ coworkers } & ENU & 51 & 4.1422 & .71808 & .10055 \\
\hline & WNU & 65 & 4.1038 & .73539 & .09121 \\
\hline \multirow[t]{2}{*}{ communication } & ENU & 51 & 3.9118 & .93250 & .13058 \\
\hline & WNU & 65 & 3.8308 & .80052 & .09929 \\
\hline \multirow[t]{2}{*}{ Nature of work } & ENU & 51 & 4.2010 & .74334 & .10409 \\
\hline & WNU & 65 & 3.7846 & .68590 & .09508 \\
\hline
\end{tabular}

ENU= Eurasian National University named after Gomilov, WNU=Woman National Unibersity, N=Number

From table 5 we can see that teachers in ENU are slightly more satisfied than the teachers in WNU, for example in payment level (ENU: $M=3.1824$, WNU: $M=3.0962$ ), supervision (ENU: $\mathrm{M}=3.8873$, WNU: $\mathrm{M}=3.7308$ ), contingent rewords (ENU: $\mathrm{M}=3.8186$, WNU: $\mathrm{M}=3.4193$ ), nature of work (ENU: $\mathrm{M}=4.2010$, WNU: $\mathrm{M}=3.7846$ ).

Table 8 job satisfaction in gender

\begin{tabular}{lccccc}
\hline \multirow{2}{*}{ gender } & & $\mathrm{N}$ & Mean & Std & Std. Error Mean \\
& & & & & .19696 \\
& $\mathrm{M}$ & 22 & 2.8295 & .92385 & .08041 \\
\cline { 2 - 6 } & $\mathrm{F}$ & 95 & 3.1974 & .78372 & .16065 \\
\hline promotion & $\mathrm{M}$ & 22 & 3.4205 & .75351 & .08080 \\
& & & & & \\
& $\mathrm{~F}$ & 95 & 3.5079 & .78758 & \\
\hline
\end{tabular}




\begin{tabular}{|c|c|c|c|c|c|}
\hline \multirow[t]{2}{*}{ Supervision } & $\mathrm{M}$ & 22 & 3.7955 & .63451 & .13528 \\
\hline & $\mathrm{F}$ & 95 & 3.7974 & .85854 & .08808 \\
\hline \multirow[t]{2}{*}{ Fringe benefits } & $\mathrm{M}$ & 22 & 3.1591 & .75258 & .13913 \\
\hline & $\mathrm{F}$ & 95 & 3.4711 & .76480 & .07847 \\
\hline \multirow[t]{2}{*}{ Contingent rewords } & $\mathrm{M}$ & 22 & 3.2500 & .82736 & .17639 \\
\hline & $\mathrm{F}$ & 95 & 3.6605 & .80219 & .08230 \\
\hline \multirow[t]{2}{*}{ Operating Procedures } & $\mathrm{M}$ & 22 & 3.2841 & 1.15288 & .24580 \\
\hline & $\mathrm{F}$ & 95 & 3.7605 & 1.15081 & .11807 \\
\hline \multirow[t]{2}{*}{ coworkers } & $\mathrm{M}$ & 22 & 4.3295 & .80691 & .17203 \\
\hline & $\mathrm{F}$ & 95 & 4.0763 & .69771 & .07158 \\
\hline \multirow[t]{2}{*}{ Communication } & $\mathrm{M}$ & 22 & 3.7273 & 1.02036 & .21754 \\
\hline & $\mathrm{F}$ & 95 & 3.9000 & .81410 & .08353 \\
\hline \multirow[t]{2}{*}{ Nature of work } & $\mathrm{M}$ & 22 & 4.3068 & .80524 & .17168 \\
\hline & $\mathrm{F}$ & 95 & 3.8921 & .70016 & .07184 \\
\hline
\end{tabular}

$\mathrm{N}=$ Number, $\mathrm{M}=$ Male, $\mathrm{F}=$ Female, $\mathrm{Std}=$ standard deviation

As we can see table 6, female are more satisfied than male in satisfaction of benefit (Male: $\mathrm{M}=3.1591, \mathrm{Std}=0.65258$, Female: $\mathrm{M}=3.4711$, $\mathrm{Std}=0.76480$ ), payment (Male: $\mathrm{M}=2.8295$, $\mathrm{Std}=092385$, Female: $\mathrm{M}=3.1974$, Std=0.78372), Contingent reword (Male: $\mathrm{M}=3.2500$, $\mathrm{Std}=0.82736$, Female: $\mathrm{M}=3.6605$, $\mathrm{Std}=0.80219$ ), Communication (Male: $\mathrm{M}=3.7273$, Std=1.02036, Female: $M=3.9, \mathrm{Std}=0.81410$ ), Male are more satisfied than female in nature of work (Male: $\mathrm{M}=4.3068$, Female: $\mathrm{M}=3.8921$ ), coworkers (Male: $\mathrm{M}=4.3295$, Female: $\mathrm{M}=4.0763)$.

Table 9 Faculty job satisfaction

\begin{tabular}{cccccc}
\hline Faculty & & $\mathrm{N}$ & Mean & Std Deviation & Std. Error Mean \\
\hline Pay & & & & .75022 & .07997 \\
& & & & & .14799 \\
& $\mathrm{HL}$ & 29 & 2.5862 & .79697 & .08119 \\
\hline Promotion & $\mathrm{FL}$ & 88 & 3.6051 & .76162 & .13733 \\
\cline { 2 - 5 } & $\mathrm{H}$ & 29 & 3.1466 & .73956 & .09187 \\
\hline Supervision & FL & 88 & 3.7898 & .86177 & .12707 \\
\hline & $\mathrm{H}$ & 29 & 3.8190 & .68432 & .07337 \\
\hline
\end{tabular}




\begin{tabular}{|c|c|c|c|c|c|}
\hline & $\mathrm{H}$ & 29 & 2.8966 & .71188 & .13219 \\
\hline \multirow[t]{2}{*}{ Contingent rewords } & $\mathrm{FL}$ & 88 & 3.7955 & .79016 & .08423 \\
\hline & $\mathrm{H}$ & 29 & 2.9397 & .52037 & .09663 \\
\hline \multirow[t]{2}{*}{ Operational procedures } & $\mathrm{FL}$ & 88 & 4.0227 & 1.04472 & .11137 \\
\hline & $\mathrm{H}$ & 29 & 2.6256 & .79203 & .14708 \\
\hline \multirow[t]{2}{*}{ Coworkers } & $\mathrm{FL}$ & 88 & 4.0256 & .71322 & .07603 \\
\hline & $\mathrm{H}$ & 29 & 4.4224 & .67844 & .12598 \\
\hline \multirow[t]{2}{*}{ Communication } & $\mathrm{FL}$ & 88 & 3.8920 & .83631 & .08915 \\
\hline & $\mathrm{H}$ & 29 & 3.7931 & .92873 & .17060 \\
\hline \multirow[t]{2}{*}{ Nature of work } & $\mathrm{FL}$ & 88 & 3.9063 & .73823 & .07870 \\
\hline & $\mathrm{H}$ & 29 & 4.1638 & .70482 & .13088 \\
\hline
\end{tabular}

$\mathrm{FL}=$ foreign language, $\mathrm{H}=$ history, $\mathrm{H}=$ number

As the table 9 illustrates, there are big differences in two faculties in comparing the teachers job satisfaction level, teachers in the faculty of foreign language are more satisfied than the teachers in faculty of history in payment (FL: $\mathrm{M}=3.3068, \mathrm{H}: \mathrm{M}=2.5862$ ), fringe benefits (FL: $\mathrm{M}=3.5824, \mathrm{H}: \mathrm{M}=2.8966$ ), contingent rewords ( $\mathrm{FL}: \mathrm{M}=3.7955, \mathrm{H}: \mathrm{M}=2.9397$ ), however, the teachers in the faculty of history have higher satisfaction than the teachers in faculty of foreign language in nature of work $(\mathrm{H}: \mathrm{M}=4.1638, \mathrm{FL}: \mathrm{M}=3.9063)$, and coworkers $(\mathrm{H}$ : $\mathrm{M}=4.4224$, FL: $\mathrm{M}=4.0256)$.

Table 10 Job satisfaction with different faculties

\begin{tabular}{|c|c|c|c|c|c|c|c|c|c|}
\hline & & \multirow[t]{4}{*}{$\mathrm{N}$} & \multirow[t]{4}{*}{ Mean } & \multirow{4}{*}{$\begin{array}{l}\text { Std. } \\
\text { Deviation }\end{array}$} & \multirow{4}{*}{$\begin{array}{l}\text { Std. } \\
\text { Error }\end{array}$} & $95 \%$ & confidence & \multirow{4}{*}{ Min } & \multirow[t]{4}{*}{ Max } \\
\hline & & & & & & \multicolumn{2}{|c|}{ interval for Mean } & & \\
\hline & & & & & & Lower & Upper & & \\
\hline & & & & & & Bound & Bound & & \\
\hline \multirow[t]{3}{*}{ Pay } & ML & 84 & 3.2173 & .78270 & .08540 & 3.0474 & 3.3871 & 1.25 & 5.25 \\
\hline & $\mathrm{PhD}$ & 29 & 2.9138 & .85376 & .15854 & 2.5890 & 3.2385 & 1.25 & 4.50 \\
\hline & Pro & 4 & 2.8125 & 1.21407 & .60703 & .8806 & 4.7444 & 1.00 & 5.25 \\
\hline \multirow[t]{3}{*}{ Promotion } & ML & 84 & 3.5952 & .75541 & .08242 & 3.4313 & 3.7592 & 2.00 & 5.75 \\
\hline & $\mathrm{PhD}$ & 29 & 3.2414 & .82235 & .15271 & 2.9286 & 3.5542 & 2.00 & 4.75 \\
\hline & Pro & 4 & 3.1250 & .52042 & .26021 & 2.2969 & 3.9531 & 2.50 & 3.75 \\
\hline \multirow[t]{2}{*}{ Supervision } & ML & 84 & 3.8304 & .75367 & .08223 & 3.6668 & 3.9939 & 1.00 & 6.00 \\
\hline & $\mathrm{PhD}$ & 29 & 3.6293 & .93927 & .17442 & 3.2720 & 3.9866 & 1.00 & 5.50 \\
\hline
\end{tabular}




\begin{tabular}{|c|c|c|c|c|c|c|c|c|c|}
\hline & Pro & 4 & 4.3125 & 1.14337 & .57168 & 2.4931 & 6.1319 & 3.50 & 6.00 \\
\hline \multirow[t]{3}{*}{ Fringe benefits } & ML & 84 & 3.5208 & .70199 & .07659 & 3.3685 & 3.6732 & 2.00 & 5.00 \\
\hline & $\mathrm{PhD}$ & 29 & 3.1293 & .85987 & .15967 & 2.8022 & 3.4564 & 1.50 & 4.50 \\
\hline & Pro & 4 & 3.1875 & .42696 & .21348 & 2.5081 & 3.8669 & 2.75 & 3.75 \\
\hline Contingent & ML & 84 & 3.6607 & .80627 & .08797 & 3.4857 & 3.8357 & 1.75 & 5.25 \\
\hline \multirow[t]{2}{*}{ reword } & $\mathrm{PhD}$ & 29 & 3.2759 & .73904 & .13724 & 2.9947 & 3.5570 & 2.00 & 5.00 \\
\hline & Pro & 4 & 4.1875 & 1.14337 & .57168 & 2.3681 & 6.0069 & 2.75 & 5.50 \\
\hline \multirow{3}{*}{$\begin{array}{l}\text { Operating } \\
\text { procedures }\end{array}$} & ML & 84 & 3.7411 & 1.05243 & .11483 & 3.5127 & 3.9695 & 1.50 & 5.75 \\
\hline & $\mathrm{PhD}$ & 29 & 3.3621 & 1.37838 & .25596 & 2.8378 & 3.8864 & 1.50 & 5.50 \\
\hline & Pro & 4 & 4.4375 & 1.39007 & .69503 & 2.2256 & 6.6494 & 3.00 & 5.75 \\
\hline \multirow[t]{3}{*}{ Coworkers } & ML & 84 & 4.1935 & .75085 & .08192 & 4.0305 & 4.3564 & 2.50 & 6.00 \\
\hline & $\mathrm{PhD}$ & 29 & 3.9397 & 62198 & .11550 & 3.7031 & 4.1762 & 2.75 & 6.00 \\
\hline & Pro & 4 & 4.0000 & .70711 & .35355 & 2.8748 & 5.1252 & 3.00 & 4.50 \\
\hline \multirow[t]{3}{*}{ Nature of work } & ML & 84 & 3.9702 & .78138 & .08526 & 3.8007 & 4.1398 & 2.00 & 5.75 \\
\hline & $\mathrm{PhD}$ & 29 & 4.0172 & .55874 & .10376 & 3.8047 & 4.2298 & 2.75 & 5.75 \\
\hline & Pro & 4 & 3.6250 & .96825 & .48412 & 2.0843 & 5.1657 & 2.75 & 5.00 \\
\hline \multirow[t]{3}{*}{ Communication } & ML & 84 & 3.8929 & .82896 & .09045 & 3.7130 & 4.0728 & 2.25 & 6.00 \\
\hline & $\mathrm{PhD}$ & 29 & 3.7586 & .94841 & .17611 & 3.3979 & 4.1194 & 2.25 & 5.50 \\
\hline & Pro & 4 & 4.1250 & .77728 & .38864 & 2.8882 & 5.3618 & 3.50 & 5.25 \\
\hline
\end{tabular}

$\mathrm{ML}=$ Master level, Pro=Professor, Min= Minimum, $\mathrm{Max}=$ Maximum

Teachers job satisfaction level is also depend on teachers' individual differences like educational qualifications, As we can see in table 11 teachers with lower qualification (master degree teachers), are more satisfied than $\mathrm{PhD}$ and Professors in payment (ML: $\mathrm{M}=3.2173, \mathrm{SD}=0.78270, \mathrm{Min}=1.25, \mathrm{Max}=5.25 ; \mathrm{PhD}: \mathrm{M}=2.9138, \mathrm{SD}=0.85376, \mathrm{Min}=1.25$,

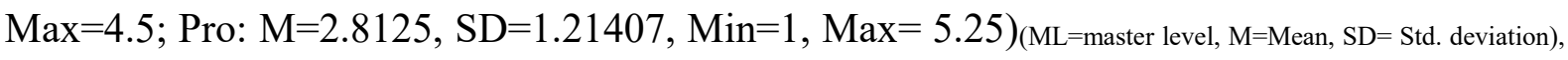
promotion (ML: $\mathrm{M}=3.595, \mathrm{SD}=0.75541, \mathrm{Min}=2, \mathrm{Max}=5.75$; $\mathrm{PhD}: \mathrm{M}=3.2414, \mathrm{SD}=0.82235$, $\operatorname{Min}=2, \operatorname{Max}=4.75$; Pro: $M=3.1250, \mathrm{SD}=0.52042$, $\mathrm{Min}=2.50, \mathrm{Max}=5.75)$, fringe benefits $(\mathrm{ML}$ : $\mathrm{M}=3.5208, \mathrm{SD}=0.70199, \mathrm{Min}=2, \mathrm{Max}=5, \mathrm{PhD}: \mathrm{M}=3.1293, \mathrm{SD}=0.85987, \mathrm{Min}=1.5, \mathrm{Max}=4.5$; Pro: $\mathrm{M}=3.1875, \mathrm{SD}=0.42696$, $\mathrm{Min}=2.75, \mathrm{Max}=3.75$ ). However, if the qualification of the teachers is getting high the teachers are more satisfied in supervision, contingent rewords, operating procedures and communication processes. 
Table 11 leadership style in different faculties

\begin{tabular}{llllll}
\hline Faculty & & $\mathrm{N}$ & Mean & Std Deviation & Std, Error De \\
\hline Transformational leadership & $\mathrm{FL}$ & 92 & 2.8762 & .65774 & .06857 \\
\cline { 2 - 6 } & $\mathrm{H}$ & 31 & 2.6075 & .53972 & .09694 \\
\hline Transactional leadership & $\mathrm{FL}$ & 92 & 3.5625 & .60612 & .06461 \\
\cline { 2 - 6 } & $\mathrm{H}$ & 31 & 2.8161 & .56404 & .10474 \\
\hline Lassiz-faire leadership & $\mathrm{FL}$ & 92 & 2.4624 & .67020 & .06987 \\
\cline { 2 - 6 } & $\mathrm{H}$ & 31 & 2.7174 & .51408 & .09233 \\
\hline
\end{tabular}

$\mathrm{FL}=$ foreign language, $\mathrm{H}=$ history, $\mathrm{N}=$ number

The information in table 11 shows that faculty of foreign language is using transactional leadership style $(\mathrm{M}=3.5625, \mathrm{SD}=0.60612)$, while the faculty of history is using transformational $(\mathrm{M}=2.8762, \mathrm{DS}=0.53972)$, transactional $(\mathrm{M}=2.8161, \mathrm{M}=0.56404)$, and lassis-faire leadership style $(\mathrm{M}=2.7174, \mathrm{SD}=0.51408)$.

Table 12 Multiple Linea Regression for Job Satisfaction (N=123)

\begin{tabular}{|c|c|c|c|c|c|}
\hline & \multicolumn{2}{|c|}{ Unstandardized } & \multirow{2}{*}{$\begin{array}{l}\text { Standardized } \\
\text { coefficients }\end{array}$} & \multirow{3}{*}{$\mathrm{t}$} & \multirow{3}{*}{ sig } \\
\hline & \multicolumn{2}{|c|}{ Coefficients } & & & \\
\hline & B & Std. Error & Beta & & \\
\hline Transformational & 0.091 & 0.048 & 0.135 & 1.896 & 0.060 \\
\hline Transactional & 0.462 & 0.042 & 0.714 & 11.006 & 0.000 \\
\hline Laisses-faire & -0.048 & 0.049 & -0.071 & -0.989 & 0.325 \\
\hline Constant & 1.981 & .201 & & 9.876 & .000 \\
\hline
\end{tabular}

$\mathrm{R}^{2}=0.529$

For understanding of the question about which leadership style is influencing teachers' job satisfaction, I created a multiple linear regression model. From the finding we can see that (see Table 12), three different leadership styles are influencing teachers job satisfaction in different ways; there is very weak influence from Transformational leadership style to teachers' job satisfaction $(\mathrm{B}=0.091, \mathrm{t}=1.896$, $\mathrm{Sig}=0.060)$, while the Transactional leadership style influence teachers job satisfaction level is high $(\mathrm{B}=0.462, \mathrm{t}=11.006, \mathrm{Sig}=0.000)$, but the Lasses-faire leadership style has negative influence for teachers job satisfaction level $(\mathrm{B}=-$ 0.048, $\mathrm{t}=-0.989$, Sig=0.325). When a leader is using transactional leadership style, he/she can increase his/her employees' satisfaction level by 0.462 . 

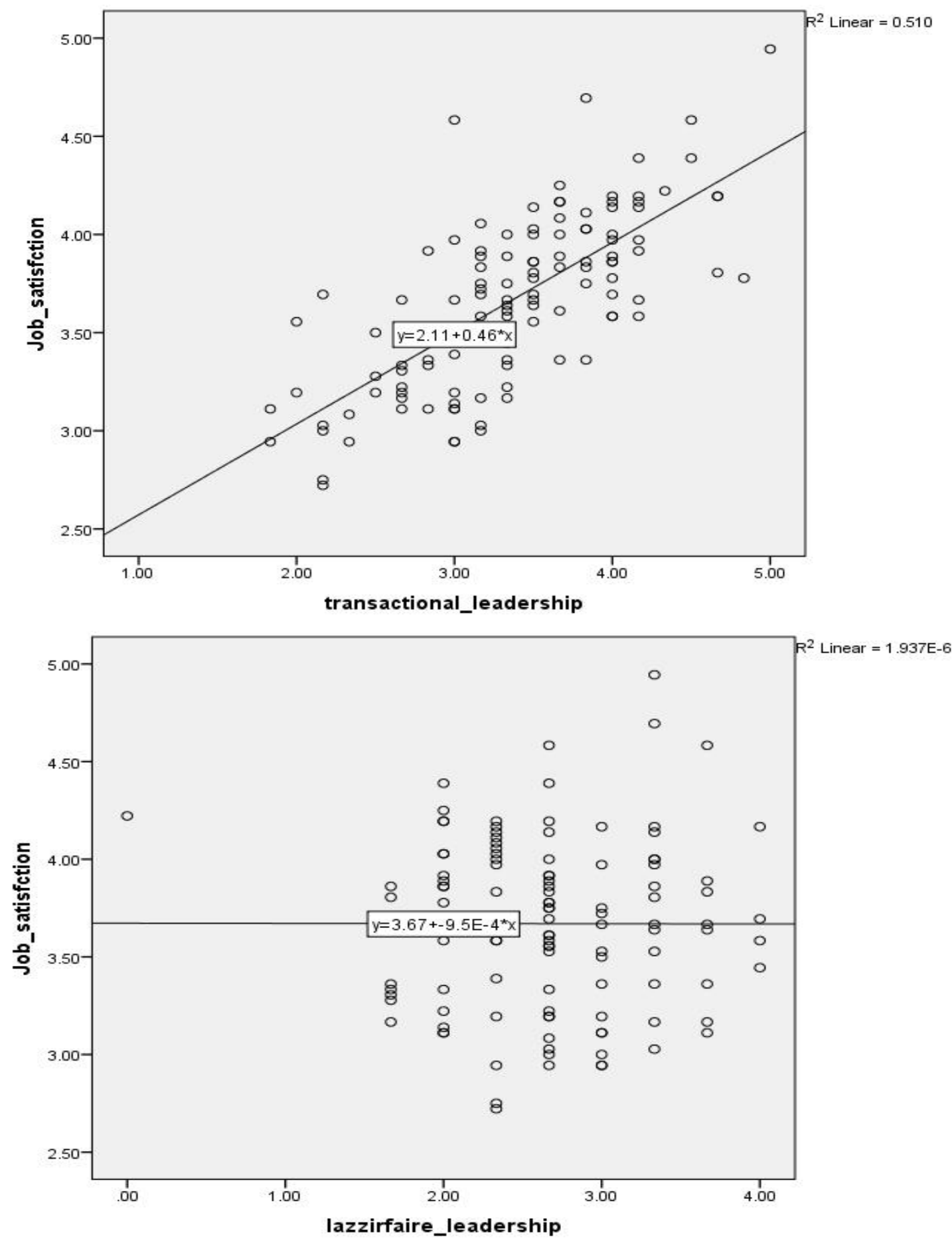


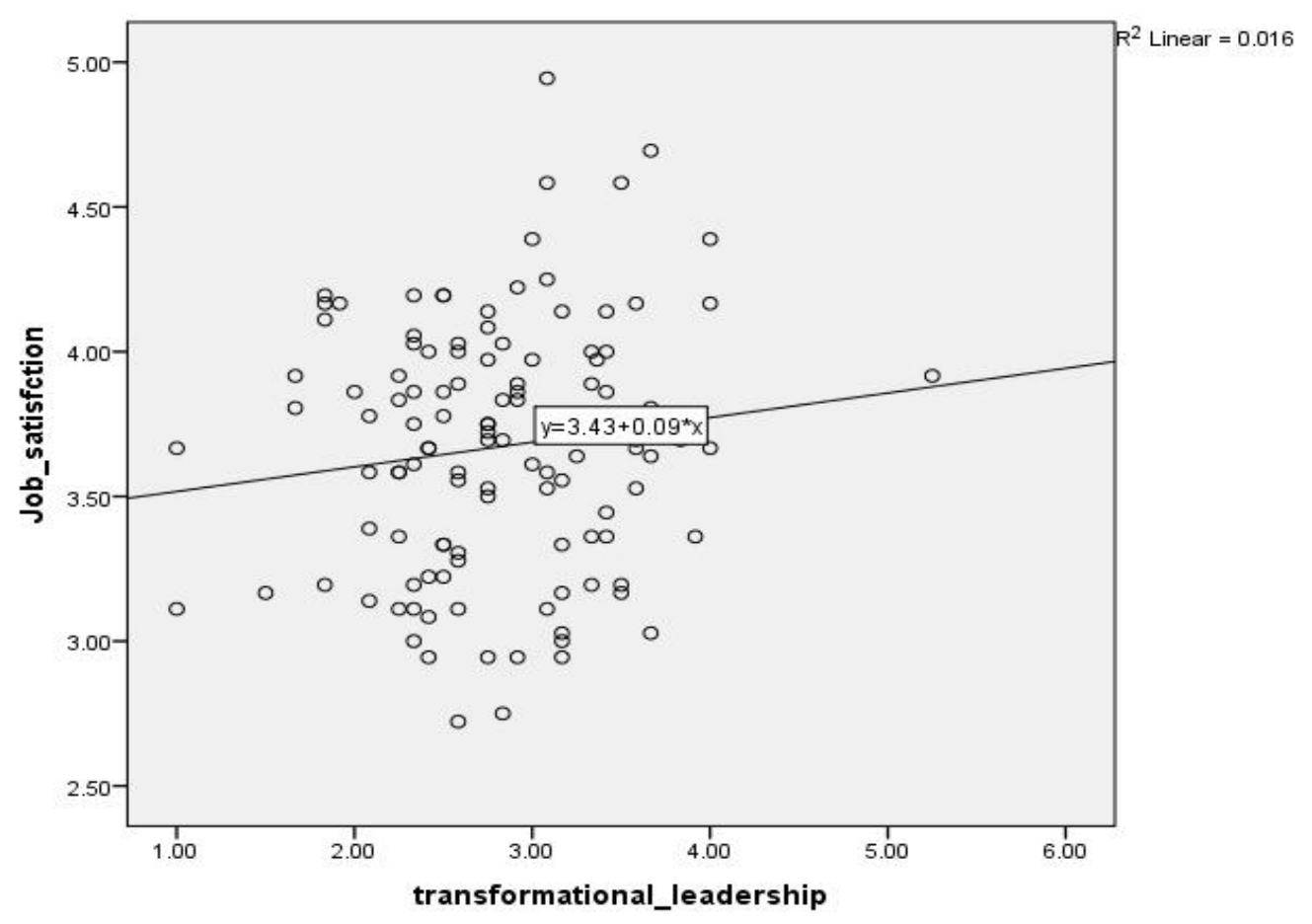

\section{Discussion}

This paper explored the relationship between faculty leaders (dean, HOD) and faculty teachers job satisfaction with in the two national universities in Kazakhstan with the participations of teachers $(\mathrm{N}=117)$ and leaders $(\mathrm{N}=7)$ in two different faculties. The collection of data is supporting the idea of there is a strong correlation between leaders' leadership style and teachers job satisfaction. Transactional leadership style is the most used by faculty leaders (Dean, HOD) in two universities (Kazakh National Woman Training University and L.N. Gumilov Eurasian National University) in Kazakhstan. Transformational, Transactional leadership style have positive impact on teachers' job satisfaction level, while the Laisses-faire leadership style has negative influence. Evans (2001) argues that teachers job satisfaction is influenced by many factors like salary, education policy and reforms also conditions of service. He also established that leadership emerged as a key attitudesinfluencing factors, since it shapes teachers work context and has the capacity through policy implementation and decision making, to enable or constrain and to determine individuals' proximity to their ideal job.

Studies about influence of leadership style on job satisfaction imply the fact that the school principal style play an important role in influencing teachers' job satisfaction (Skrapits, 1986; Rosenholtz, 1989; Andermann et al., 1991; Billingstley, 1993; Lashbrook, 1997; Lok and Crawfor, 1999; Schultz and Teddlie, 1999; Methrotra, 2005; Sharma and Jyoti, 2006; Cerit, 
2009). Job satisfaction proved not only to be dependent on leadership style, but also teachers' individual differences like gender, marital stats. Gender is also one of the factors for influence teachers job satisfaction level. From this paper we understand that female teachers are more satisfied than male teachers, same result can be seen in other scholars' findings (Sharma \& Jyoyi, 2009; Wagner \& French, 2010). However, other researchers found out opposite result (Bilimoria et al., 2006; Callister, 2006; Hult et al., 2006). Except gender differences scholars also identified that the teachers with young age are less satisfied and more likely to leave from their working places than olders (Viel-Ruma et al., 2010). Marital status can be also considerate for the correlation between teachers' job satisfaction level. According to the studies marriage has positive relation with teachers' job satisfaction (Sabharwal \& Corley, 2009; Sharma \& Jyoti, 2009). A study about teacher organizational commitment at secondary School in Addis Ababa showed that there is a correlation between teachers' marital stage and their commitment level and conclude that marriage teachers are more committed than single teachers (Endale, 2019), same result is found in this paper. Teachers academical level is the one of the main factor which influence teachers job satisfaction level. One of the findings showed that teachers with lower qualification (master level) are tend to be more satisfied than the $\mathrm{PhD}$ holders and professors. This finding is in accordance with the findings of Abdullah \& Parasuraman (2009), while another study found that there was a noticeable difference between graduate and non-graduate teachers job satisfaction level (Nguni \& Sleegers,2009). From this finding we can say that the teachers with lower level of education are more satisfied (payment, promotion, fringe benefit) in their job, this maybe for these teachers there is an opportunity to update their level in the context. On the other hand, the teachers with $\mathrm{PhD}$ and professor level are more satisfied than the less qualified teachers in supervision, contingent rewords, operating procedures and communication. This maybe high qualified teachers are more likely to be a leader with good communication skills. Another interesting finding is that there is big differences in two faculties (foreign language and history) in comparing the teachers job satisfaction level, teachers in the faculty of foreign language are more satisfied than the teachers in faculty of history in payment (FL: $\mathrm{M}=3.3068, \mathrm{H}: \mathrm{M}=2.5862$ ), fringe benefits (FL: $\mathrm{M}=3.5824, \mathrm{H}$ : $\mathrm{M}=2.8966$ ), contingent rewords ( $\mathrm{FL}: \mathrm{M}=3.7955, \mathrm{H}: \mathrm{M}=2.9397)$, however, the teachers in the faculty of history have higher satisfaction than the teachers in faculty of foreign language in nature of work $(\mathrm{H}: \mathrm{M}=4.1638, \mathrm{FL}: \mathrm{M}=3.9063)$, and coworkers $(\mathrm{H}: \mathrm{M}=4.4224$, FL: $\mathrm{M}=4.0256$ ). Allison (2017) found that there is a strong correlation between racial groups. Asian faculty are more dissatisfied with the appreciation and recognition they receive for 
their scholarly work (Mean=2.65), while white faculty are most satisfied with this appreciation and recognition (Mean=3.29).

\section{References}

[1] Abdullah, M., Uli, J., \& Parasuraman, B. (2009). Job satisfaction among secondary school teachers. Journal Kemanusiaan, 13,11-18.

[2] Al-Omari, A. (2007), Leadership Style of Department Chairs at the Hashemite University. Journal Of Education and Psychology Sciences, 8(3), 7-24.

[3] Al-Omari, A. (2008). The Relationship between Leadership styles of Hashemite University Department Chairs and job Satisfaction as Reported by Department Faculty Members. University of Sharjah Journal for Humanities \& Social Sciences, 5(2), 101124.

[4] Armstrong, M. (2006). A Handbook of Human Resource Management Practice. (10th ed.) London: Kogan Page.

[5] Bass B.M. and Riggio, R.E. (2006). Transformational Leadership. (2nd ed.) Mahwah: Lawrence Erlbaum Associates.

[6] Bohen, S.j. and Stiles, J. (1998). Experimenting with Models of Faculty Collaboration: Factors That Promote their Success. New Directions for Institutional Research, 98(100), $39-55$.

[7] Bogler, R. (2001). The influence of Leadership Style on Teacher Job Satisfaction. Educational Administration Quarterly, 37(5),662-683.

[8] Blankenship, S. L. (2010). The consequences of transformational leadership and/ot transactional leadership in relationship to job satisfaction and organizational commitment for active duty women serving in the air force medical service (Order No. 3398823). Available from ProQuest Dissertation \& Theses Full Text: The Humanities and Social Sciences Collection. (276227724). Retrieved from http://search.proquest.com/docview/276227724?accountid=7374

[9] House, R.J. (1976). A 1976 Theory of Charismatic Leadership in Hunt, J.G and Larson L.L. (eds) Leadership: The Cutting Edge. A Symposium Held at Southern Illinois University, Carbondale. 189-207. Carbondale: Southern Illinois University press.

[10] Shah, S. (2006a). Leading Multiethnic School: a new understanding of Muslim Youth Identity. Educational Management, Administration and Leadership, 34(2), 215-237. 
[11] Bass, B.M., Avolio, B. J., Jung, D. I., \& Berson, Y. (2012). Predicting unit performance by Assessing transformational and transactional leadership. Journal Applied Psychology, 88(2), 207-218.doi:10.1037/0021-9010.88.2.207.

[12] Burns, J.M. (1978). Leadership. New York: Harper \& Row.

[13] Dale, J. C., Jr. (2012). The correlation of the perceived leadership style of middle school principals, To teacher job satisfaction and efficacy. (Order No.3546181, Liberty University). ProQuest Dissertation and Thesis, 197. Retrieved from http://search.proquest.com/docview/1238003178?accountid=7374.

[14] Duyar, I., Gumus, S., \& Mehmet, S.B. (2012). Multilevel analysis of teacher work attitudes. The International Journal of Education Management, 27(7), 700-719. Doi:10.1108/IJEM-092012-0107.

[15] Eyal, O., \& Roth, G., (2011). Principals' leadership and teachers' motivation. Journal of Educational Administration 49(3), 256-275. Doi:10.1108/09578231111129055

[16] Ingersoll,R. M. (2003). The teacher shortage: Myth or reality. Education Horizons, 81(3), 146-152. Retrieved from eric.ed.gov/?id=EJ665621.

[17] Klassen, R., \& Chiu, M. (2010). Effects on teachers' self-efficacy and job satisfaction: Teacher gender, Year of experience, and job stress. Journal of Educational Psychology, 102, 741-756. doi: 10.1037/a0019237

[18] Nadim, M., Muhammad, S.C., Massod, N.K., \& Riaz, T. (2012). Effects of motivational factors On teachers' job satisfaction: A study on public sector degree colleges of Punjab, Pakistan. The journal of Commerce, 4(4), 25. Retrieved from http://search.proquest.com/docview/1221264426? Accounted $=7374$

[19] Peacock, J. S. (2014). Science instructional leadership: The role of the department chair. $\begin{array}{llll}\text { Science } & \text { Educator, } & \text { 23(1), } & \text { Retrieved }\end{array}$ http://lopes.idm.oclc.org/login?url=http://search.proquest.com.lopes.idm.oclc.org/docvie w/1552464392? Accounted $=7374$

[20] Sawchuk, S. (2012). Principals criticized on teacher-retention decisions. Education Week, 31(37), 17. Retrieved from www.edweek.org. 